\title{
Structural Efficiency and Robustness Evolution of the US Air Cargo Network from 1990 to 2019
}

\author{
Changsong Zhao $\mathbb{D D}^{1}$ and Chunliang Xiu $\mathbb{D}^{2}$ \\ ${ }^{1}$ School of Business Administration, Northeastern University, Shenyang 110169, Liaoning, China \\ ${ }^{2}$ Jangho Architecture College, Northeastern University, Shenyang 110169, Liaoning, China \\ Correspondence should be addressed to Chunliang Xiu; xiuchunliang@mail.neu.edu.cn
}

Received 28 August 2021; Revised 7 October 2021; Accepted 5 November 2021; Published 20 November 2021

Academic Editor: May T. Lim

Copyright (c) 2021 Changsong Zhao and Chunliang Xiu. This is an open access article distributed under the Creative Commons Attribution License, which permits unrestricted use, distribution, and reproduction in any medium, provided the original work is properly cited.

\begin{abstract}
As the US air cargo network (USACN) becomes a crucial part of the economy, it is pivotal to understand the structural evolution of the network and how it would be affected by unexpected events. We investigated the network structure, efficiency, and robustness of the USACN from 1990 to 2019 due to targeted attacks based on complex network theory from a dynamic and spatiotemporal perspective. Our results suggest that the USACN has enhanced robustness. Moreover, we find that attacks based on betweenness centrality are the most effective way to cause a collapse compared with attacks based on degree and closeness centrality. In addition, airports of the USACN have formed an increasing number of communities with geographical ties, and airports in the noncontiguous regions are more vulnerable than other communities in the lower 48 states. Further, we discover that the average path lengths have increased, and the overall efficiency has decreased from 0.7 to 0.4 due to the dependency of the hub-and-spoke structure. This paper complements previous studies on the dynamic structure evolution of air cargo networks through the lens of complex network theory with spatial-temporal data.
\end{abstract}

\section{Introduction}

Air transportation contributes to a large proportion of the global economy, and it has drawn increasing attention from scholars during recent years. According to [1], though the air cargo industry transports $1 \%$ of the total volume, it contributes to $35 \%$ of the total world trade value. Despite its importance, there has been a lack of literature on the resilience of air transportation networks as opposed to other types of networks such as railroad, subway, and power grid. Multiple real-world scenarios in the past have suggested that disruptions such as severe weather events, terrorist attacks, or global pandemics could bring catastrophic effects for the airline industry and regional and global economics for both air passenger and cargo networks. Therefore, it is of paramount value to assess the robustness of the air transport network when facing either random errors or targeted attacks. The most recent case of the COVID-19 global pandemic has had a devastating impact on the air transportation industry and the global economy beginning from April 2020 with the travel restrictions grounding flights and blocking connections amongst cities. Both air passenger and cargo demand plummeted $69.7 \%$ and $20 \%$, respectively, while the air cargo demand decreased slower due to the global shortage of medical supplies and equipment. Moreover, according to [1], 44 million jobs in the aviation industry were affected, in addition to a net loss of 118.5 billion USD in the air passenger sector, and 173 billion USD in the cargo sector. Consequently, it is critical to study the mechanism of air transportation network structures, efficiency, and robustness when facing similar disruptions. Graph theory and complex network theory are the main tools scholars employed to investigate the characteristics of the air transportation network [2-5]. In summary, previous studies have examined topology using cross-sectional data, and therefore, there is a lack of literature in academia that explores the air transport network from a temporal perspective.

In this study, we aim to (1) explore the topological evolution of the USACN through the lens of spatialtemporal view and (2) empirically examine the spatial 
properties including overall network efficiency, robustness, and community structures. The rest of the paper is structured as follows. Section 2 briefly encapsulates the previous literature. Section 3 describes the methodologies. In section 4, we portray the spatial-temporal evolution of the USACN from 1990 to 2019. Section 5 describes the characteristics of robustness and efficiency. Finally, in Section 6, we conclude this study with some discussion.

\section{Literature Review}

A wide spectrum of literature has investigated the air transportation network in the past, which can be categorized into three main groups. The first group describes the topological features of the air transportation network, and the connections to the cities. Branching from this line of research, the second group of studies has also explored network robustness and accessibility for both air cargo and passenger networks. The third group of literature focuses majorly on the economic implications of the air transportation network, covering a wide spectrum of topics ranging from the concept of Aerotropolis [6] to the business model such as LCC and FSC [7], carbon emission [8], gravity model and granger causality test [9] to investigate the economic connections between airports and local and regional economics, ticket fare based on the air transportation network structure, and fuel efficiency for passenger and air cargo market. In addition to the policy in the air transportation industry, which is the fundamental driving force for the development.

2.1. Network Topography. In the research of the topology of the network structure, scholars have extensively employed complex network theory to assess the network structure on varieties of geographical scales such as EU, US, India, Brazil, and China [10-19]. Further, air passenger and cargo have been explored separately, though there is a nonproportional number of studies centered on the air passengers as opposed to cargo counterpart. One reason for such unbalance in academia is that the economic contribution of the air cargo industry has drawn increasing attention only during recent years. Most of the previous studies have identified the small-world and scale-free properties on a variety of geographical scales [20] [21]. The authors of [22, 23] analyzed the world airport network and uncovered the fact that the traffic between airports is subject to the capacity of the airports, and by simulating a global epidemic outbreak, they conclude that the air transport network could accelerate the speed of transmission. The authors of [3] performed an analysis on the global air cargo transport network using flight frequency and discovered that air cargo networks have a unique structure because of their operational differences. The authors of [24] analyzed the air cargo network structures using the case of FedEx and UPS and further illustrated the differences between cargo and passenger counterparts.
2.2. Robustness and Resilience. The robustness of a system refers to the ability to maintain its original functionality after attacks or disasters [25]. It has been of paramount interest due to the vital role in our networked systems such as energy [26-28], water system [29-32], telecommunications [33, 34], and transportation. Previous studies in the field of transportation have looked at multiple types of transportation networks including metro/subway system [35-37], highway and road systems [38-40] [41], and marine system [42-44].

Given the growing economic contribution of the air transportation network, the robustness and resilience have drawn an increasing number of investigations to assess the robustness of the air transportation network weighted [45-47]. Geographically speaking, scholars inspected the robustness of air transportation networks in the $\mathrm{EU}[17,48]$, the US [46, 49], and China [50, 51]. Dunn and Wilkinson [52] compared two strategies for increasing the resilience of air traffic networks: an adaptive reconfiguration strategy and a permanent rerouting strategy. [49, 52] examined the resilience of the US national airport network based on network science and proposed that adaptively rerouting and recovery strategies would increase the robustness. The authors of [53] evaluated the network performance and revealed that airports' robustness is affected by different key factors during large-scale disruptive events. Regardless, most of the preceding studies suggest that the air transport network robustness is related to the structural formation, which is determined by political, economic incentives of airline companies, and local (regional) policies. The authors of [7] discovered that EU, China, and North American airlines began the transition from the single model to a hybrid model to increase network efficiency.

2.3. Air Transportation Liberalization Policy and Economic Development. The third group of literature explores the driving force for air transportation development-policy and management strategies-which has drawn a renewed interest on a variety of geological scales, with plenty of empirical evidence in multiple geographical regions, such as the US-Caribbean [54], EU [55], Africa [56], Asia [57], and the Middle East [58]. All the above studies have confirmed that air transport liberalization has brought economic properties to those regions. The authors of [59] examines 184 countries and discovered that liberalization has fostered the increase of passenger flow. From a temporal perspective, [60] suggested that the long-term OECD air transportation demand depends on the level of liberalization policies. Additionally, the prevalence of the LCC (low-cost carriers) across the globe has tremendously stimulated local and regional economic growth [7, 20, 61-65].

2.4. Research Gap and Contribution in the Literature. Our contributions to the existing studies are as follows. First, our work investigates the dynamic structures, efficiency, and robustness evolution of the air cargo network from a spatialtemporal perspective, which is lacking in the previous literature. Second, we remove edges instead of nodes as the attack strategy because of its practicality, since even in the extreme case of the COVID-19 global pandemic, most 
airports still operate with limited capacity instead of completely shut down. Third, we assess the dynamic evolution of the USACN structure, efficiency, and robustness using longterm data from a spatial-temporal viewpoint, which fills the gaps of research that focus specifically on the air cargo network structures.

\section{Methodology and Datasets}

3.1. Data Selection and Preparation. The data in this study comes from the US Bureau of Transportation Statistics (https:// www.bts.gov/) website. We focus on the domestic operations of the US air cargo operations from 1990 to 2019. The transportation data is organized into individual tables on an annual basis, which contains the $O D$ information regarding passengers, cargo, distances, carriers, etc., and the total size of the dataset is about $1 \mathrm{~Gb}$. In this paper, we model the USACN as $O D$ networks with the airports representing the nodes and routes that connect the nodes as edges. The raw dataset is subsequently processed to build the US airport network for each year. Most of the previous literature has considered the air transportation network as unweighted and undirected, and we take similar approaches as $[3,66,67]$, where the networks are modeled as directed and weighted. The rationale behind such depiction is that an unweighted network could present bias caused by the prevalence of network topology. In processing the data, we combine the passenger-cargo with the all-cargo hauls and assume that all the passengers carry $23 \mathrm{~kg}$ of luggage in the combination of the freight and mail on board and consider this as the total volume that one aircraft can carry. To illustrate the temporal changes in both networks, we perform analyses of cargo flow and classify airports using community structures in $1990,2000,2010$, and 2019, respectively. Second, we examine the efficiency for the overall and largest community structures each year. Third, we calculate robustness to explore the structure impacts of each attack strategy.

3.2. Centrality Indices and Network Fragmentation. The degree of an airport $i$ represents the number of flights/routes connected to it, which determines its connections to the rest of the network; in other words, the airports with higher degree values connect with more airports.

Betweenness centrality $(b c)$ measures the probability of a node that is on the shortest paths of the other two nodes, reflecting the node's transit capability. In other words, a node with a higher $b c$ is more likely to be a connecting hub, where $\sigma_{s t}(i)$ is the total number of the shortest paths between nodes $s$ and $t$ that pass through $i$, and $\sigma_{s t}$ is the total number of the shortest paths between $s$ and $t$ that pass through $i$, and it is expressed as [68]

$$
c_{B}(i)=\sum_{s \neq t \neq i}^{n} \frac{\sigma_{s t}(i)}{\sigma_{s t}} .
$$

Closeness centrality (cc) measures the average distance from a given starting node to all other nodes in the network, expressed as

$$
C_{c}=\frac{(N-1)}{\sum_{i \neq j, i \in G} d_{i j}}
$$

3.3. Network Community Structures. This community structure refers to vertices or nodes that cluster into several groups in which nodes are more concentrated within each group than among groups. In air transportation networks, community structures can detect the geographical clusters of airports by measuring the intensity of the flow amongst cities, and the spatial pattern of airports distribution. Specifically, we extract the fragmentation and structure of the air cargo network by measuring the largest community structure $(l c s)$ based on the modularity class $m[69,70]$. The higher the modularity value, the tighter the connection of the network. Furthermore, the decomposition of the network into a set of subgraphs $C_{i}$, which has a unique value of MR (modularity resolution) associated with this partition, can be measured as follows [71]. The modularity value $M R \in(-1,1)$, which measures the density of links inside communities as compared to links between communities. In a weighted network, it is defined as

$$
\mathrm{MR}=\frac{1}{2 m} \sum_{i, j}\left[A_{i j}-\frac{k_{i} k_{j}}{2 m}\right] \delta\left(c_{i}, c_{j}\right),
$$

where $a_{i j}$ represents the cargo volume between two airports $i$ and $j, k_{i}=\sum_{j} a_{i j}$ is the total cargo volume of the flights of the airport $i, c_{i}$ represent the airport $i$ is located in, the $\delta$ function $\delta(u, v)$ is 1 if $u=v$, and 0 otherwise, and $m=(1 / 2) \sum_{i j} a_{i j}$. By measuring MR, we can identify both the number and the size of the subgraphs and reveal the geographical characteristics.

3.4. Robustness Assessment. In air transportation, robustness is defined as the ability to remain functional from the loss of critical airports and routes [72, 73]. In this paper, we assess the network robustness by removing nodes based on their centrality values and obtain the rest iteratively until there is no largest connected component remaining. We constructed a Network Integrity Index $(R)$ to explore each cross-sectional data when that moment occurs. According to [74], on a weighted network, the robustness of a weighted network can be expressed as a function of the fraction of nodes (airports) removed. Hence,

$$
R=\frac{1}{N} \sum_{i=1}^{N} C(F)
$$

where $N$ is the total number of airports in the network at a given moment and $C(F)$ represents the largest connected community structure when $F=f n$ edges are removed from the network. Consequently, the removal of key routes between airports in the network would dramatically reduce the resilience of the network. Subsequently, the lower the $R$ value, the less the resilience of the network.

3.5. Network Efficiency. The network efficiency is defined as the average value of the reciprocal of the path lengths 
between any two nodes in a network [75]. In this context, we use the Dijkstra algorithm to calculate the shortest paths between nodes. Mathematically, when nodes $i$ and $j$ are not connected, $d_{i j}=+\infty$, and therefore, $\left(1 / d_{i j}\right)=0$. In this study, we define the mean value of efficiency between all nodes in the network, which is expressed as

$$
E(G)=\frac{1}{N(N-1)} \sum_{i \neq j}\left(\frac{1}{d_{i j}}\right) .
$$

Here, $N$ is the number of nodes in the current network. In general, the shorter the path lengths of the route (edge), the more efficient the network. $E \in(0,1)$, where 0 represents a completely disconnected graph and 1 represents a fully connected graph under the ideal condition.

\section{Evolution of USACN Structure and Properties}

This study employs Gephi, QGIS, ArcGIS, and python for processing data and representing findings, and the data is available online, and therefore the process is replicable. The findings of this study are presented as follows.

4.1. Complex Network Properties. We discover that the USACN has grown more complex during the study period. As seen in Table 1, both the nodes and edges number increased drastically. Further, the network density has increased by $157 \%$ from 7 to 18 , which suggests that, on average, one node connects to 7 other nodes in 1990 and 28 other nodes in 2019. Additionally, the average path length has increased from 1.814 to 2.343 , indicating that, on average, the air cargo needs to travel for a long distance from one airport to another. Specifically, $L=2.343$ in 2019 suggests that, in the 279-airport system, cargos can reach any other airport by two transfers on average. The high clustering coefficient indicates that an airport's topological neighbors are also likely to be connected. Furthermore, we discover that the average path length $L$ is relatively short and close to the value of the random network $L_{\text {rand }}$, and the average clustering coefficient $C \gg C_{\text {rand }}$, indicating that USACN has small-world $[76,77]$ and scale-free properties.

We have also explored the evolution of the in-degree $\left(k_{\text {in }}\right)$ and out-degree $\left(k_{\text {out }}\right)$. Figure 1 suggests that both $k_{\text {in }}$ and $k_{\text {out }}$ conform to a power-law distribution, which indicates that there are hierarchical structures for both in the USACN. In other words, few airports with high centrality values serve as transfer hubs in the network and connect with a majority of airports. The top airports with high $k_{\text {in }}$ and $k_{\text {out }}$ include Memphis, Louisville, and Anchorage, along with passenger hubs such as Atlanta, Dallas, Chicago, and Denver because the belly-cargo still plays a vital role in transporting goods as passengers travel.

4.2. Network Topography and Community Structure. Figure 2 suggests that the USACN has expanded toward the Rocky Mountains from the coastal regions, and both networks' primary hubs are in the Southeast, Midwest, and Southwest regions, partly due to the geographical advantages of being able to reach major metropolises within a few hours after the overnight sorting process from the air cargo processing facilities. Tables $2-5$ further illustrate the shifts of top airports based on centrality rankings from the study period. The transition of key airports in the USACN is because of the crucial roles of FedEx and UPS in the US domestic air cargo industry. According to the Department of Transportation Bureau of Statistics (USDOT BTS) data, in the year 2019, before the global pandemic, the market share of FedEx and UPS combined is $69.6 \%$. Even during the global pandemic of COVID-19 in 2020 and 2021 (up to June 2021), the two integrators account for $67.7 \%$ of the total market share, which further vindicates the critical roles of FedEx and UPS in the air cargo sector.

Communities are clusters of strongly connected nodes, and the communities are interconnecting with few links.

Figure 3 portrays the evolution of the community structures of the USACN from 1990 to 2019. We discovered that the airports in each community have strong ties within their geographical vicinity. Part of the reason for such spatial formation of the network structure is to minimize the transaction cost and maximize fuel efficiency when flying amongst sorting facilities and distribution centers. Consequently, we label each community based on its geographic region. Additionally, the number of communities increased slightly from 27 (36\%) in 1990 to $42.45 \%$ in 2019 . In the meantime, the cargo volume transferred through the largest LCS has risen from $27 \%$ to $30.68 \%$, indicating that while the LCS is populous of the maximum number of airports, the emerging airports serve as transshipping hubs that play an increasingly important role in the air cargo network. The Midwest and Southeast regions have the largest community structures throughout the years, which includes the airports including both air cargo and passenger hubs such as, Atlanta, Memphis, Louisville, Dallas, Chicago, Detroit, Charlotte, Indianapolis, and Minneapolis-St. Paul.

\section{Network Efficiency and Robustness Assessment}

5.1. Network Efficiency. Contrary to previous studies, we discovered that the global efficiency $E$ was 0.7 in 1990 and decreased to 0.4 in 2019, indicating a decrease in transferability of the USACN, as shown in Figure 4 . This is because the USACN has established a hierarchical hub and spoke structure, as most of the connections amongst airports go through central hubs, sorting facilities, and regional warehouses instead of point-to-point routes. It is more economical for the integrators to consolidate their resources and facilitate the growth of the hubs to exploit the density economies.

As for each year, we adopt sequential attacks based on centrality metrics, and we recalculate the metric iteratively until the collapse of the network. We discovered that, in each year, the hub airports usually (a) have higher betweenness and degree centrality since such airports usually have high $k_{\text {in }}$ and $k_{\text {out }}$, and strong capability for transferring, such as Memphis, Chicago, and Atlanta, and (b) they have no complementary airports. In other words, when connections 
TABLE 1: USACN complex network properties from 1990 to 2019.

\begin{tabular}{ccccccccc}
\hline Year & Nodes & Edge & Average degree & Average path length & Clustering coefficient & Average distance & $L_{\text {rand }}$ & $C_{\text {rand }}$ \\
\hline 1990 & 78 & 593 & 7.178 & 1.814 & 0.503 & 0.587 & 1490.86 & 1.968 \\
1995 & 215 & 2762 & 12.847 & 1.746 & 0.097 \\
2000 & 230 & 3131 & 13.613 & 2.354 & 0.62 & 0.1465 .16 & 2.287 & 0.048 \\
2005 & 272 & 4287 & 15.761 & 2.38 & 0.542 & 858.42 & 2.068 & 0.071 \\
2010 & 262 & 3815 & 14.561 & 2.366 & 0.555 & 1016.64 & 2.042 \\
2015 & 269 & 4022 & 14.952 & 2.343 & 0.07 & 1063.21 & 2.191 & 0.056 \\
2019 & 278 & 5011 & 18.025 & & 0.549 & 1006.4 & 2.185 \\
\hline
\end{tabular}
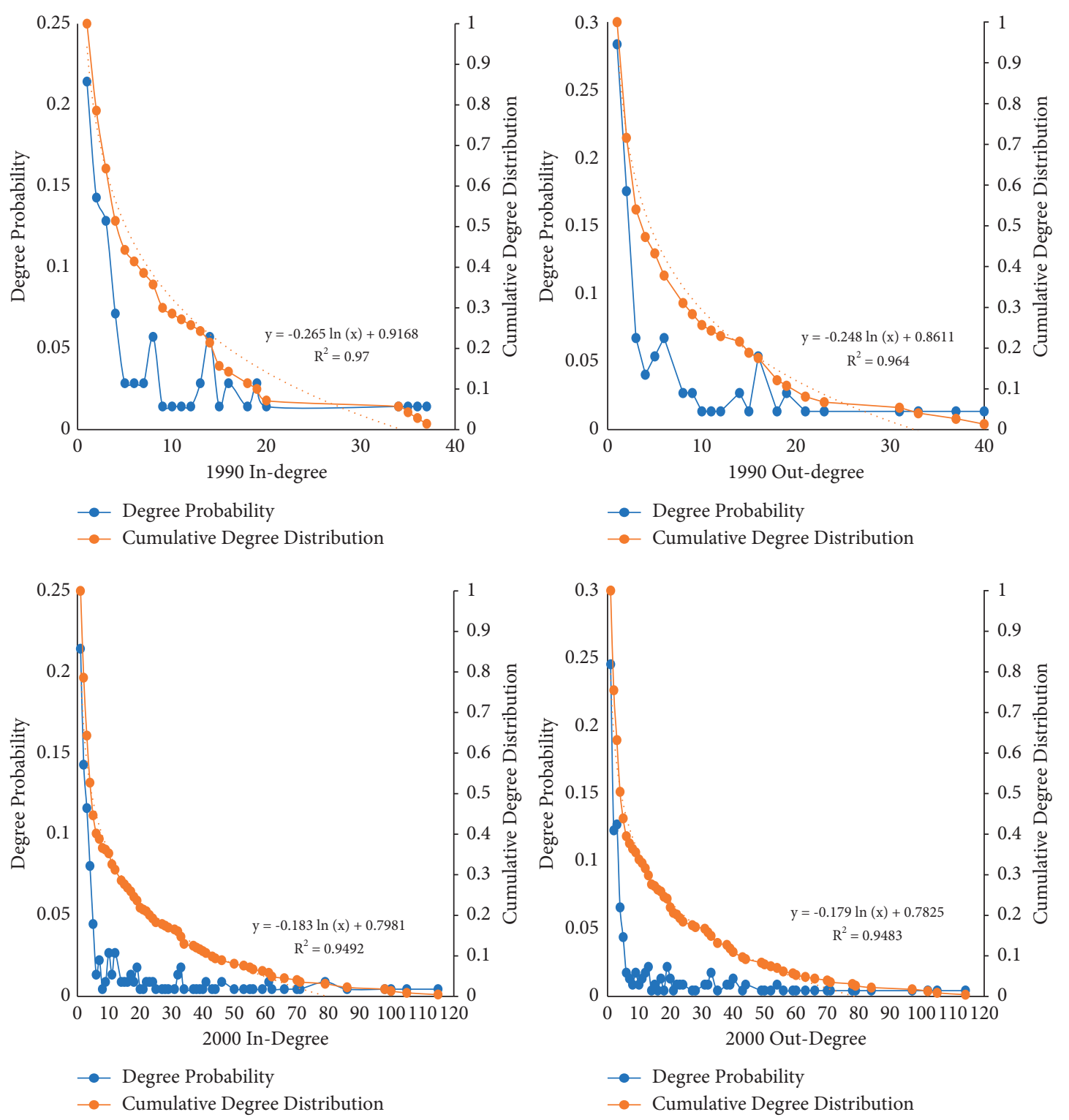

(a)

Figure 1: Continued. 

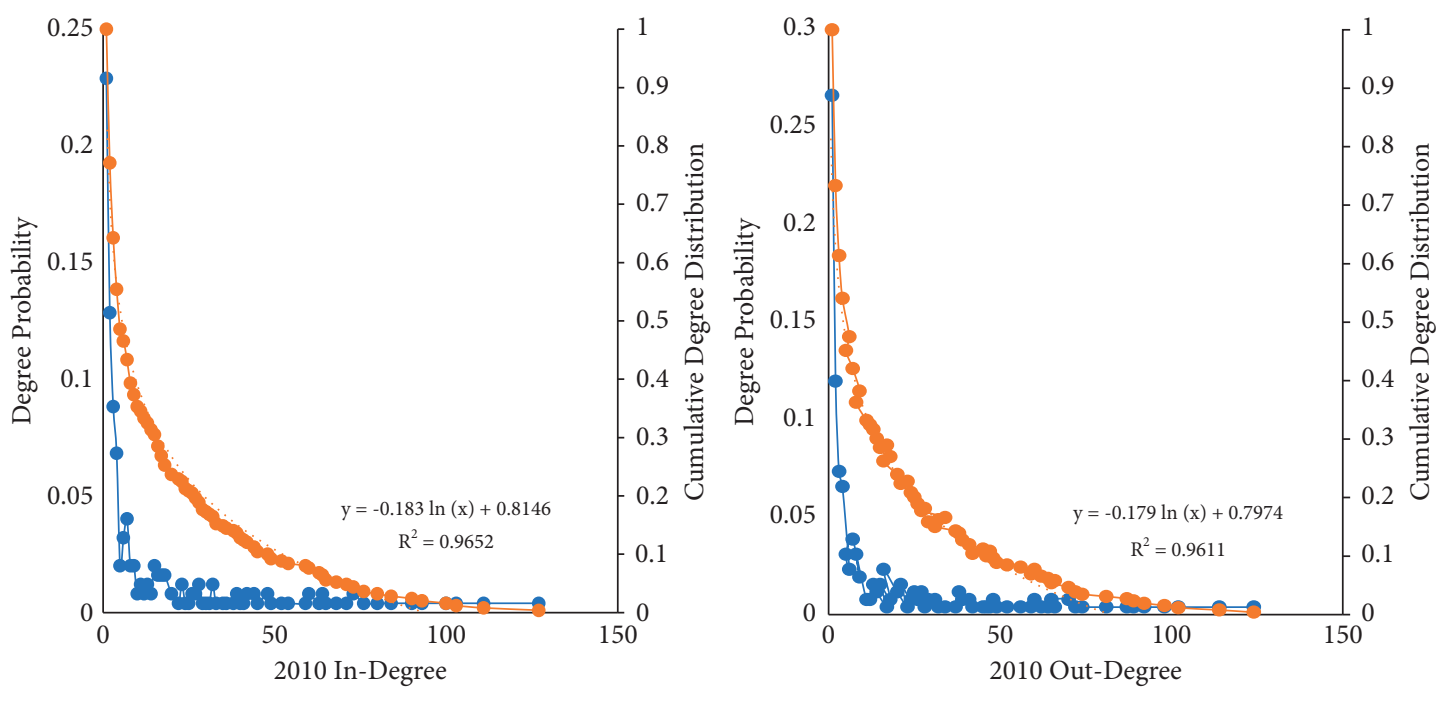

- Degree Probability

$\rightarrow$ Cumulative Degree Distribution
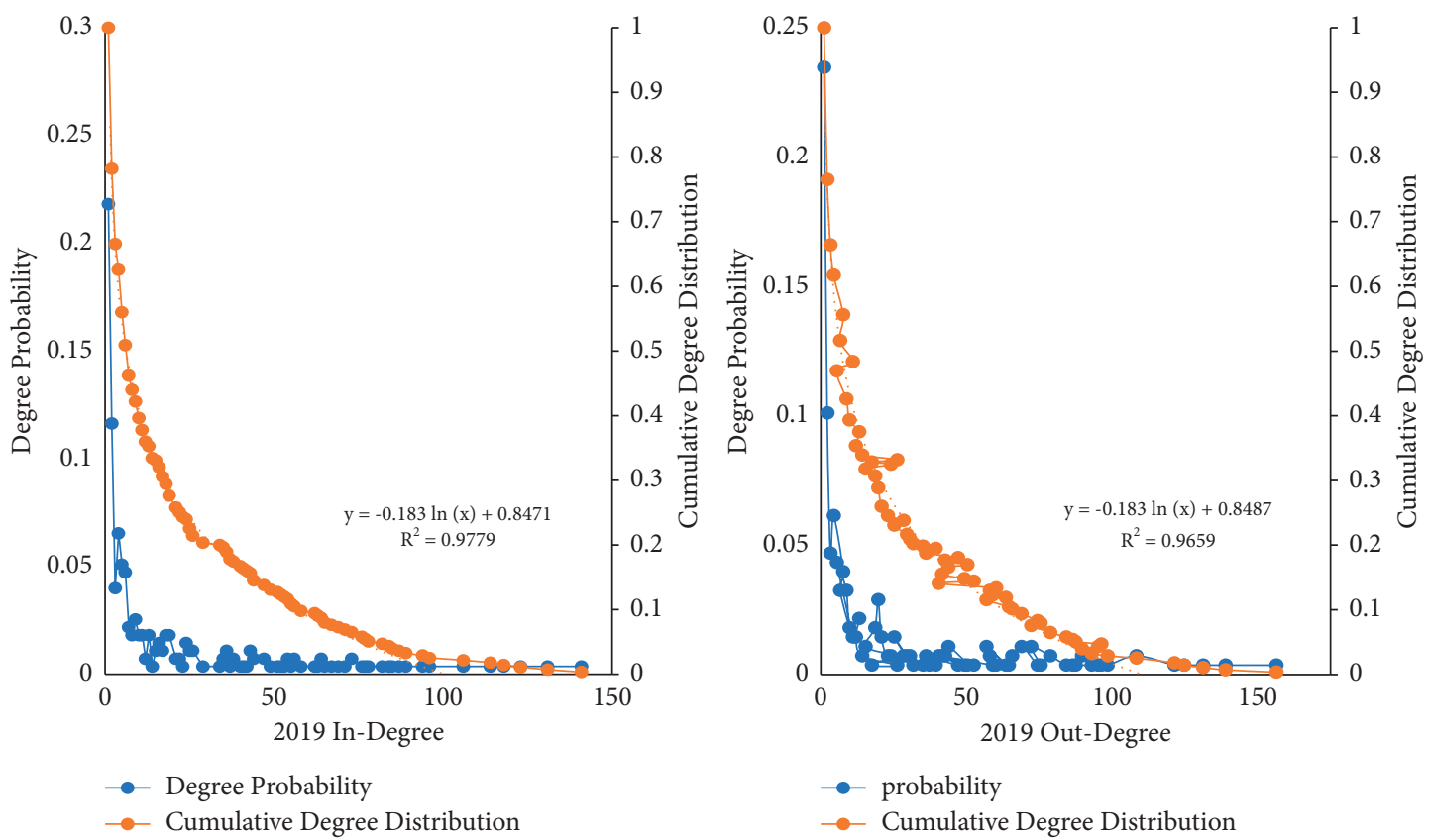

(b)

FIGURE 1: Evolution of cumulative in-degree and out-degree of the USACN from 1990 to 2019.

to those airports are closed, there are no alternatives. For example, one of the most critical airports, Memphis in the Southeast region, connects to many other smaller airports across the country, and when such an airport closes, it is difficult to find a replacement airport that is capable to handle the cargo volume. In comparison, airports with tight connections and smaller centrality values such as Philadelphia (PHL) and Newark (EWR) can be the alternative to each other, and therefore closing one would have less impact on overall network efficiency and robustness. When one such airport closes, the other can serve as a bridge to assist the cargo transfer.

5.2. Network Robustness. We assess the robustness of the USACN against the edge removal strategy and portray the spatial structure in Figures 5 and 6 . We have the following findings according to the results: 

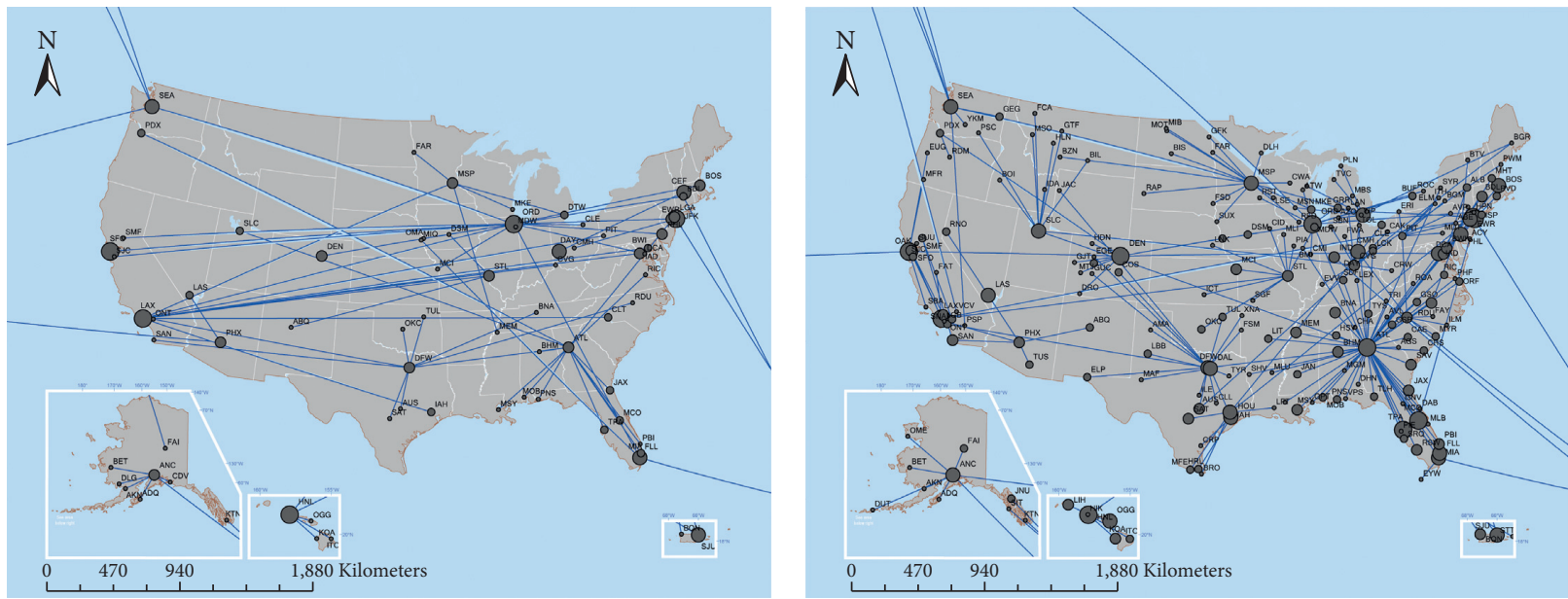

1990 US Air Cargo Network
- $100.21-231.70$
- $231.71-452.46$
- 452.47 - 688.26

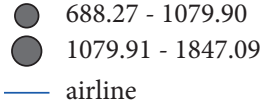

(a)

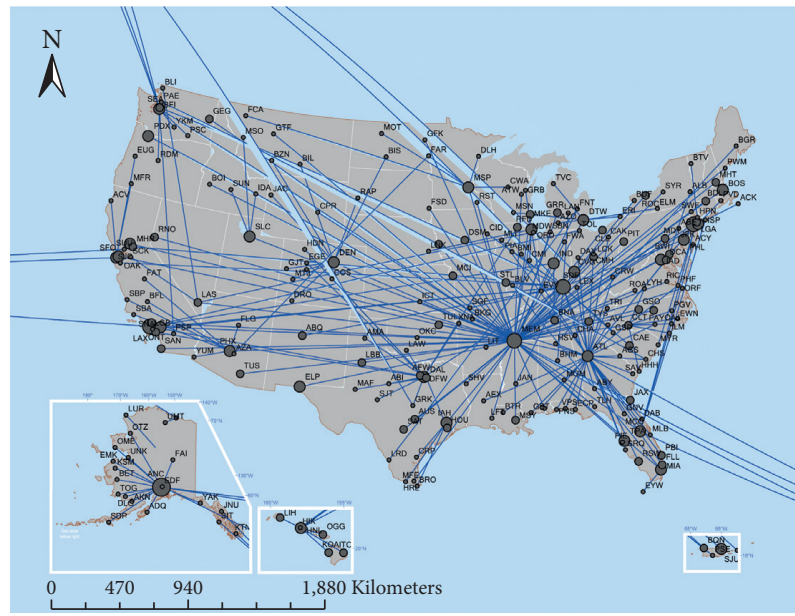

2010 US Air Cargo Network
- $100.67-830.00$
- $830.01-2034.30$
○ $2034.31-4518.91$

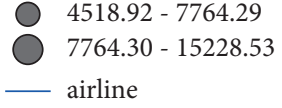

(c)
2000 US Air Cargo Network
- $100.28-403.39$
- $403.40-836.57$
- $836.58-1373.71$

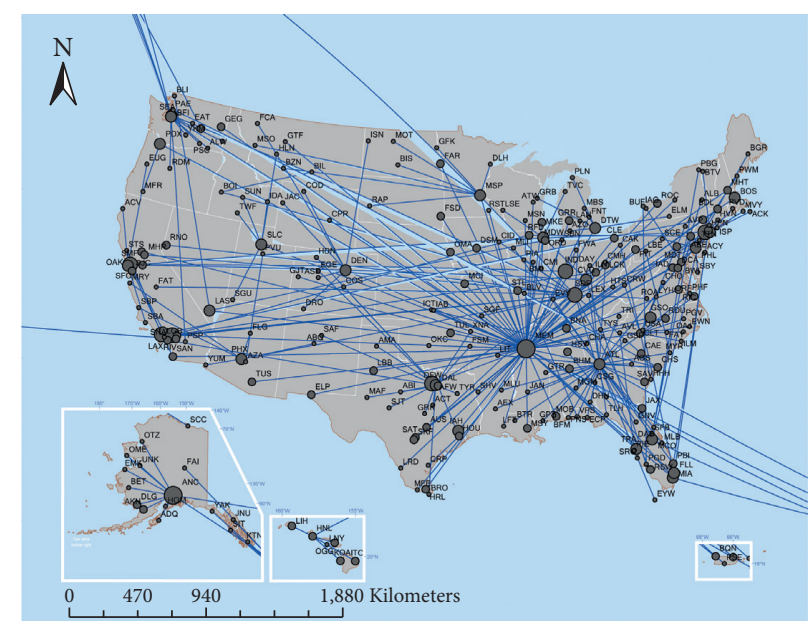

2019 US Air Cargo Network
- $102.00-828.09$
- 828.10 - 2201.74
2201.75 - 5005.54

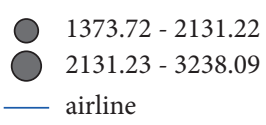

(b)

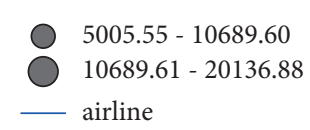

(d)

FIgURE 2: Evolution of the USACN network structure from 1990 to 2019.

TABLE 2: Rankings of the US air cargo airports based on complex network metrics in 1990.

\begin{tabular}{|c|c|c|c|c|c|c|}
\hline Rank & Airports & Degree & Airports & Closeness & Airports & Betweenness \\
\hline 1 & Chicago (ORD) & 115 & Chicago (ORD) & 0.802 & Anchorage (ANC) & 0.188 \\
\hline 2 & Dallas (DFW) & 113 & Los angeles (LAX) & 0.786 & Seattle (SEA) & 0.083 \\
\hline 3 & St. Louis (STL) & 110 & Minneapolis-St. Paul (MSP) & 0.755 & Miami (MIA) & 0.048 \\
\hline 4 & Atlanta (ATL) & 107 & Detroit (DTW) & 0.748 & Los Angeles (LAX) & 0.045 \\
\hline 5 & Los Angeles (LAX) & 102 & Dallas (DFW) & 0.740 & Chicago (ORD) & 0.041 \\
\hline 6 & Minneapolis-St. Paul (MSP) & 102 & San Francisco (SFO) & 0.733 & San Francisco (SFO) & 0.040 \\
\hline 7 & Denver (DEN) & 101 & St. Louis (STL) & 0.726 & Dayton (DAY) & 0.029 \\
\hline 8 & Pittsburgh (PIT) & 101 & Boston (BOS) & 0.726 & Minneapolis-St. Paul (MSP) & 0.025 \\
\hline 9 & Detroit (DTW) & 98 & Atlanta (ATL) & 0.720 & Hawaii (HNL) & 0.024 \\
\hline 10 & Houston (IAH) & 97 & New York (JFK) & 0.720 & New York (JFK) & 0.022 \\
\hline
\end{tabular}


TABLE 3: Rankings of the US air cargo airports based on complex network metrics in 2000.

\begin{tabular}{|c|c|c|c|c|c|c|}
\hline Rank & Airports & Degree & Airports & Closeness & Airports & Betweenness \\
\hline 1 & Dallas (DFW) & 137 & Dallas (DFW) & 0.817 & Anchorage (ANC) & 0.140 \\
\hline 2 & Atlanta (ATL) & 136 & Atlanta (ATL) & 0.817 & Seattle (SEA) & 0.079 \\
\hline 3 & Chicago (ORD) & 134 & Chicago (ORD) & 0.802 & Los Angeles (LAX) & 0.049 \\
\hline 4 & Minneapolis-St. Paul (MSP) & 130 & Minneapolis-St. Paul (MSP) & 0.802 & Dallas (DFW) & 0.031 \\
\hline 5 & St. Louis (STL) & 128 & St. Louis (STL) & 0.781 & Minneapolis-St. Paul (MSP) & 0.029 \\
\hline 6 & Detroit (DTW) & 128 & Detroit (DTW) & 0.781 & Atlanta (ATL) & 0.026 \\
\hline 7 & Cincinnati (CVG) & 127 & Houston (IAH) & 0.774 & Kansas (MCI) & 0.025 \\
\hline 8 & Houston (IAH) & 126 & Los Angeles (LAX) & 0.767 & San Francisco (SFO) & 0.024 \\
\hline 9 & Newark (EWR) & 120 & Newark (EWR) & 0.754 & Chicago (ORD) & 0.024 \\
\hline 10 & Los Angeles (LAX) & 120 & Cincinnati (CVG), & 0.748 & Philadelphia (PHL) & 0.024 \\
\hline
\end{tabular}

TABLE 4: Rankings of the US air cargo airports based on complex network metrics in 2010.

\begin{tabular}{|c|c|c|c|c|c|c|}
\hline Rank & Airports & Degree & Airports & Closeness & Airports & Betweenness \\
\hline 1 & Memphis (MEM) & 245 & Memphis (MEM) & 0.816 & Anchorage (ANC) & 0.181 \\
\hline 2 & Atlanta (ATL) & 209 & Atlanta (ATL) & 0.751 & Memphis (MEM) & 0.086 \\
\hline 3 & Chicago (ORD) & 205 & Chicago (ORD) & 0.748 & Seattle (SEA) & 0.054 \\
\hline 4 & Louisville (SDF) & 204 & Dallas (DFW) & 0.734 & Atlanta (ATL) & 0.042 \\
\hline 5 & Dallas (DFW) & 200 & Louisville (SDF) & 0.717 & Louisville (SDF) & 0.041 \\
\hline 6 & Detroit (DTW) & 183 & Houston (IAH) & 0.708 & Fairbanks (FAI) & 0.039 \\
\hline 7 & Houston (IAH) & 182 & Newark (EWR) & 0.705 & Chicago (ORD) & 0.039 \\
\hline 8 & Newark (EWR) & 178 & Minneapolis-St. Paul (MSP) & 0.696 & Dallas (DFW) & 0.033 \\
\hline 9 & Minneapolis-St. Paul (MSP) & 175 & Denver (DEN) & 0.696 & Los Angeles (LAX) & 0.026 \\
\hline 10 & Indianapolis (IND) & 174 & Los Angeles (LAX) & 0.696 & Minneapolis-St. Paul (MSP) & 0.025 \\
\hline
\end{tabular}

TABLE 5: Rankings of the US air cargo airports based on complex network metrics in 2019.

\begin{tabular}{lccccc}
\hline Rank & Airports & Degree & Airports & Closeness & Airports \\
\hline 1 & Memphis (MEM) & 243 & Memphis (MEM) & 0.796 & Anchorage (ANC) \\
2 & Chicago (ORD) & 219 & Chicago (ORD) & 0.755 & Memphis (MEM) \\
3 & Dallas (DFW) & 215 & Dallas (DFW) & 0.755 & Seattle (SEA) \\
4 & Louisville (SDF) & 213 & Louisville (SDF) & 0.737 & Chicago (ORD) \\
5 & Atlanta (ATL) & 202 & Atlanta (ATL) & 0.731 & Louisville (SDF) \\
6 & Charlotte (CLT) & 191 & Charlotte (CLT) & 0.714 & 0.058 \\
7 & Denver (DEN) & 184 & Denver (DEN) & 0.702 & Dallas (DFW) \\
8 & Philadelphia (PHL) & 174 & Phoenix (PHX) & 0.681 & Charlotte (CLT) \\
9 & Phoenix (PHX) & 173 & Philadelphia (PHL) & 0.678 & Ontario (ONT) \\
10 & Ontario (ONT) & 164 & Seattle (SEA) & 0.675 & Denver (DEN) \\
\hline
\end{tabular}

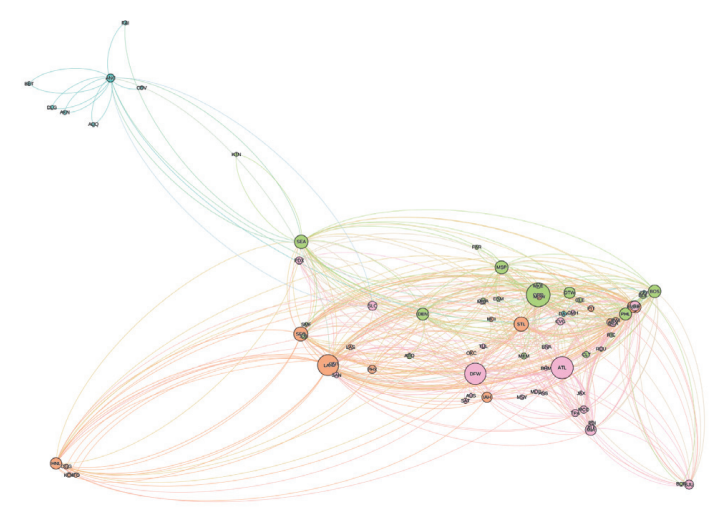

Top 3 US air cargo network community Structure 1990 Southeast, Southwest, and Northeast cs Mideast, Northeast, and Pacific cs Pacific, Southwest, and Northeast cs

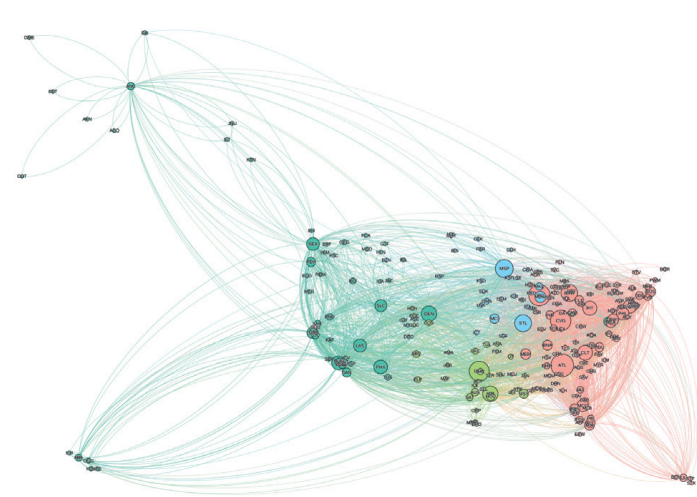

Top 3 US air cargo network community Structure 2000

$\bigcirc$ Southeast, Midwest, Noncontigious cs

O Midwest cs

Rocky Mountain, Pacific, Noncontigious cs 


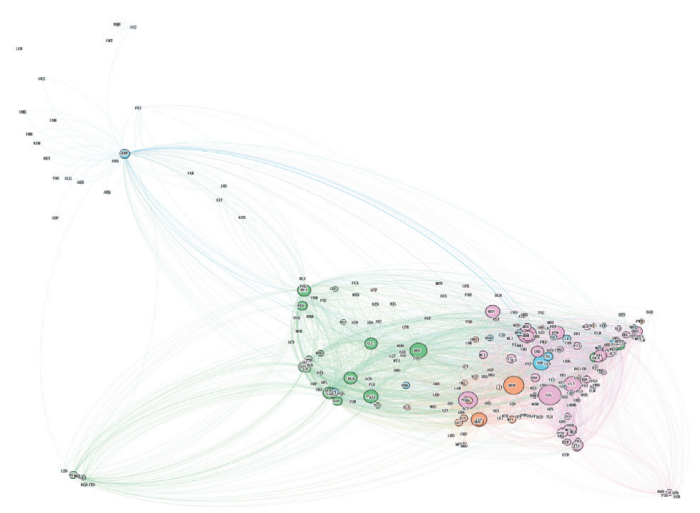

Top 3 US air cargo network community Structure 2010

O Southeast, Northeast, Southwest, Midwest cs

Southeast, Midwest cs

Pacific, Rocky Mountain, Noncontiguous cs

(c)

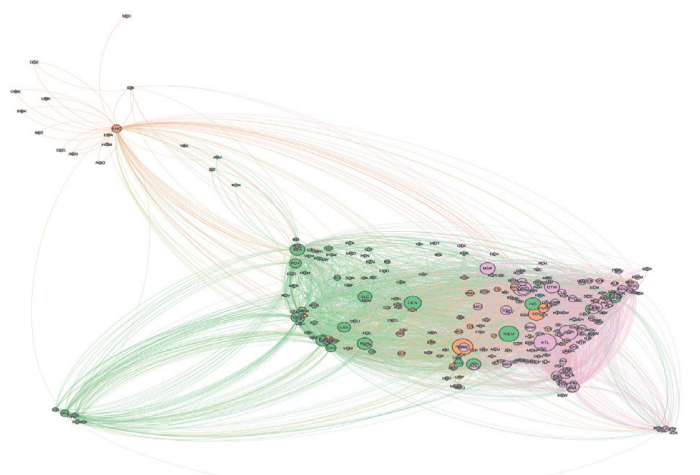

Top 3 US air cargo network community Structure 2019

O Southeast, Northeast cs

O Midwest, Southwest, Noncontiguous cs

Southeast, Midwest, Pacific, Rocky Mountain, Noncontiguous cs

(d)

FIgURE 3: Evolution of the community structures of the USACN from 1990 to 2019.
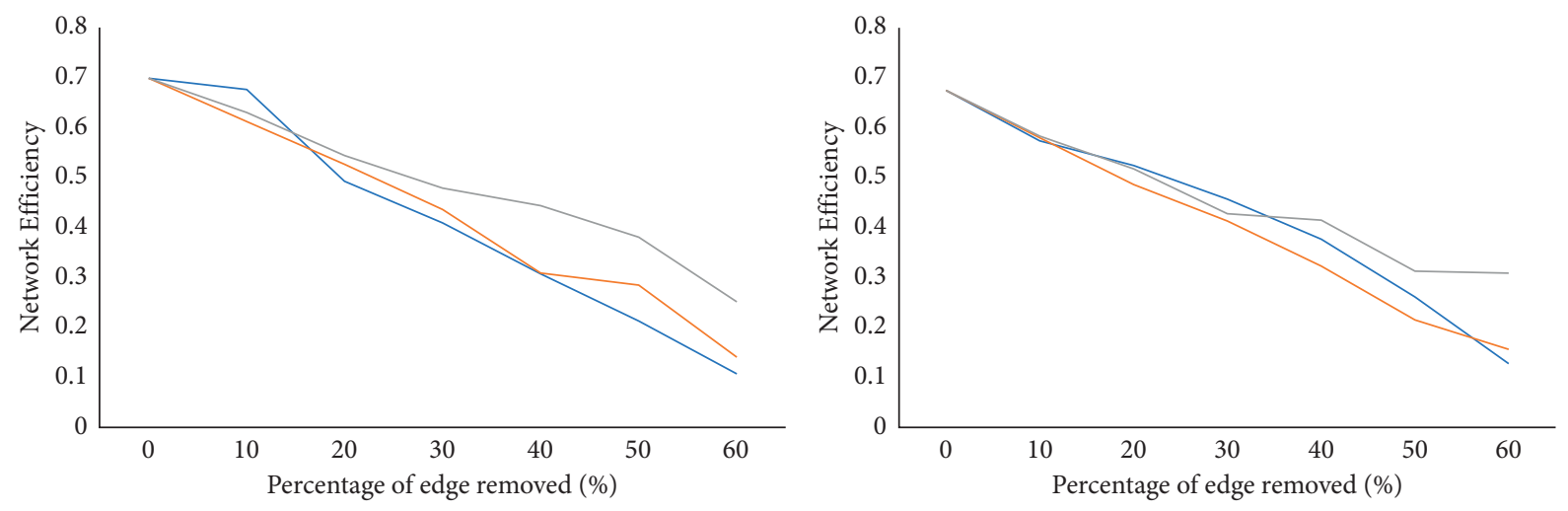

_ 1990 bc attacks

1990 degree attacks

1990 cc attacks

2000 degree attacsk

_ 2000 cc attacks
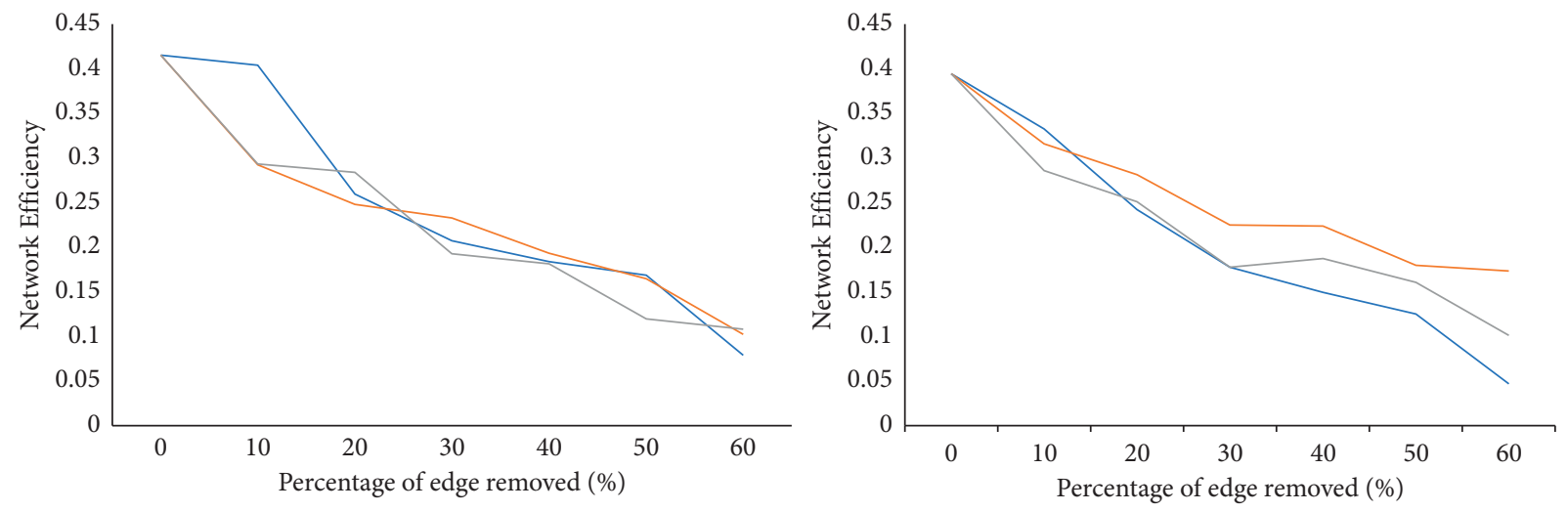

$2010 \mathrm{bc}$ attacks
_ 2010 degree attacks
$2010 \mathrm{cc}$ attacks

_ 2019 bc attacks

_ 2019 degree attacks

__ 2019 cc attacks

FIgUre 4: Evolution of the USACN efficiency under attacks based on centrality values from 1990 to 2019. 

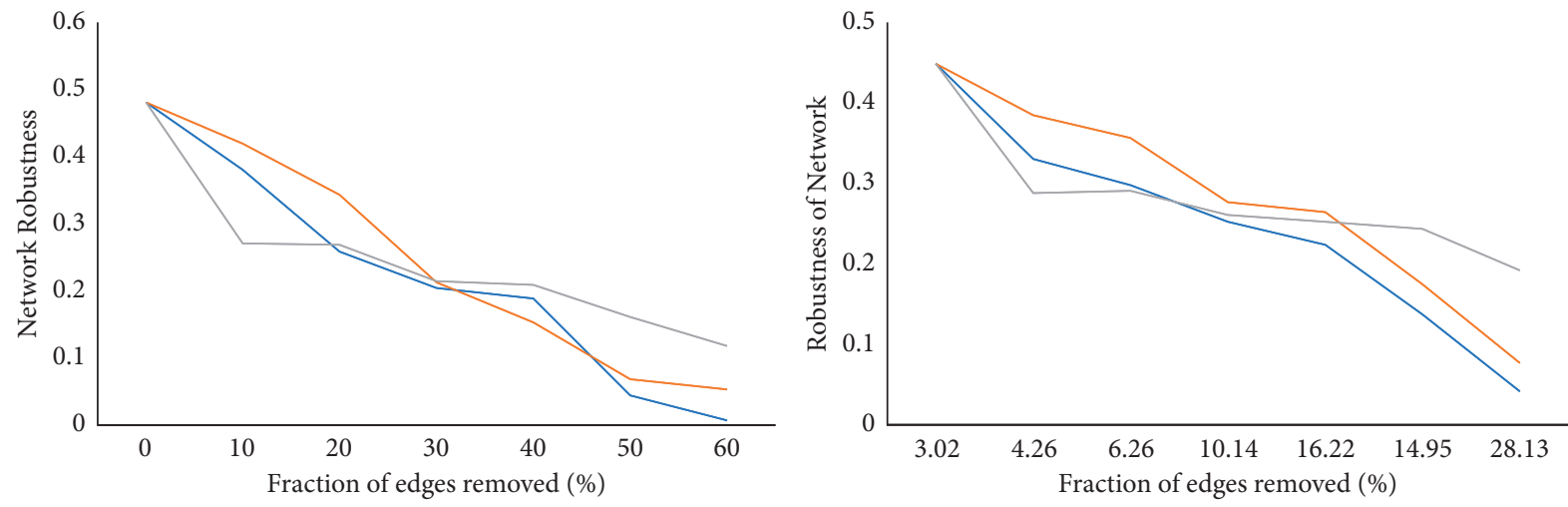

1990 Robustness bc

1990 Robustness degree

1990 Robustness cc

- 2000 Robustness bc

2000 Robustness degree

2000 Rubustness cc
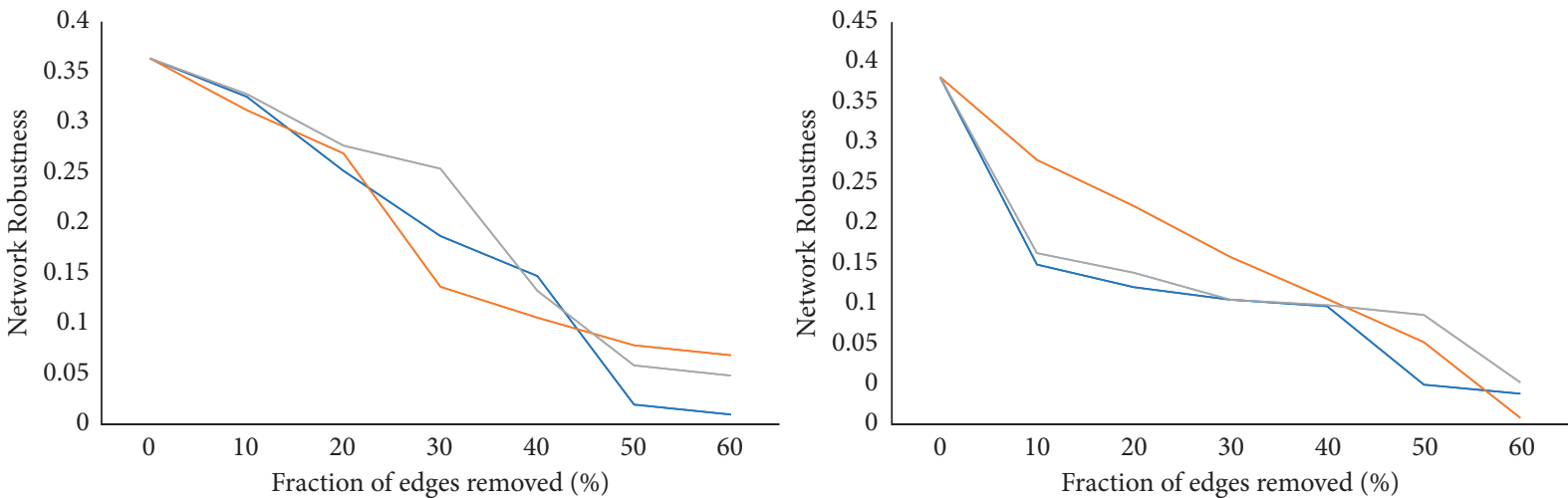

- 2010 Robustness bc

- 2010 Robustness degree

- 2010 Rubustness cc

— 2019 Robustness bc

_ 2019 Robustness degree

2019 Robustness cc

FIGURE 5: Evolution of USACN robustness under attacks based on centrality values from 1990 to 2019.

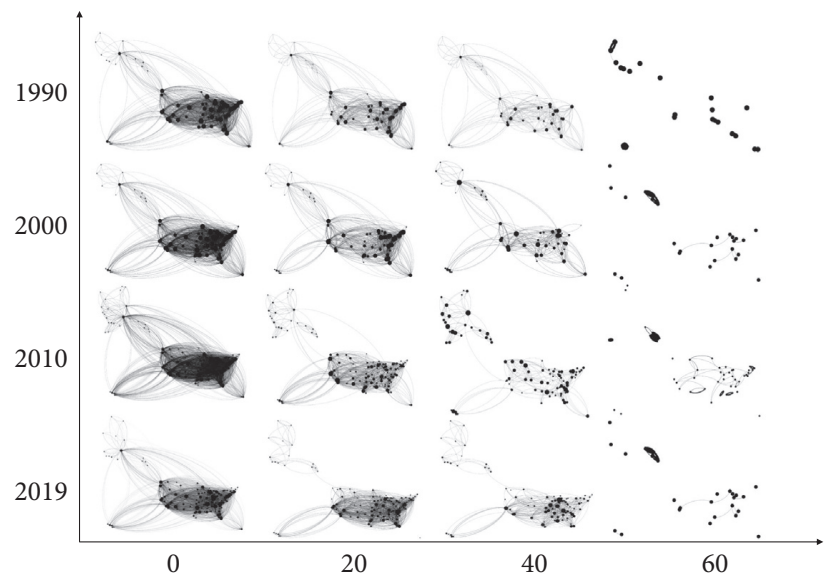

(\%)

FIGURE 6: Robustness of the USACN under attacks based on betweenness centrality from 1990 to 2019. 
(1) We find that the network integrity depends on $60 \%$ of the routes, and further removal of routes would result in the robustness dropping to nearly zero. Additionally, the attacks based on degree, $b c$, and $c c$ have similar effects on the overall network robustness. And we find that attacks on robustness are the fastest way to cause complete network paralysis.

(2) When the network is completely paralyzed, the number of routes removed has increased from 336 (out of 593) in 1990 to 3007 (5011) in 2019. This implies that the USACN has enhanced robustness as the network expanded. Additionally, the steeper slope of the decrease in robustness in Figure 5 suggests that the top 20\% of routes have strong transit capability, and removing such hubs would incur severe damage to the network integrity. As the removal of edges increases, the average clustering coefficient $C$ decreases rapidly as more nodes become less connected and more isolated. Meanwhile, the average path length $L$ decreased drastically, as the transfer of routes to hubs was removed from the network, and the network structure is composed of point-to-point connections, which further reduced the network density.

(3) Figure 6 represents the attack process based on $b c$ from 1990 to 2019. The size of the nodes represents the bc of the airports. The horizontal axis represents the network spatial structure after a $20 \%$ increment of routes removed. The vertical axis represents the years. Geographically, the longdistance route in the noncontiguous regions and remote areas are vulnerable when the flights are disconnected since they do not have alternative routes to reconnect with the rest of the network. Additionally, we discover that the network density is higher in the Southeast, Northeast, Midwest, and Southwest regions, and therefore it is relatively easier for airports in those regions to reroute their cargo to a nearby location within the geographical vicinity; thus, the $c s$ in those regions are more robust.

\section{Conclusion and Remarks}

This study examines the evolution of efficiency and robustness of the USACN in the context of a spatiotemporal perspective by portraying it as a directed and weighted network. We adopted the complex network theory to calculate the network efficiency and robustness. Our results reveal that (1) the US air cargo network grew increasingly distinctively to the air passenger counterpart. (2) The airport community structures tend to form close to their geographical proximity. Furthermore, such structure formation is primarily driven by economic reasons such as more efficient sorting facilities and lower transaction costs. With the increasing outreach of the air cargo industry, the number of communities has increased. (3) Owing to the strong dependency of the hub-and-spoke structure, the overall network efficiency decreased from 0.7 to 0.41 . Consequently, (4) attacks based on betweenness centrality values are the fastest way to cause network paralysis. Regardless, with the continuous expansion of the network, the overall robustness of the network increased over the years. The network becomes completely disconnected when 60\% (45 airports in 1990 and 162 in 2019) of routes are disabled. Geographically, the most vulnerable airports are in the noncontiguous regions such as Hawaii and Puerto Rico. Since those places have long distance and few alternative flights, therefore they would suffer most from the removal of edges, whilst, in the lower 48 states, airports in the Rocky Mountain region are more vulnerable compared with the Southeast and the coastal regions because of the low-density network. Through the analysis, we managed to extract the trunk network of the US air cargo network based on their centrality values and discovered that the evolution of the USACN is similar to other transportation networks such as railroads and subway networks, which exhibits the Matthew effect [78], indicating that new nodes tend to connect to existing high-degree nodes and conform to a hub-and-spoke network structure. Further, the US air cargo network is also subjected to strong spatial constraints and such that nodes are conterminous in space and form a spatial adjacent community structure, though air cargo is less restrained by space, with an average distance of $1000 \mathrm{~km}$ throughout the years, which translates to 2-hour flight from source to destination and can cover most of the air cargo integrators' sorting facilities.

In addition to the empirical findings, we also propose several possible managerial insights as follows. First, the hub-and-spoke network structures for both passengers and cargo networks need to be scrutinized by policymakers and investors in the new era of the global economy, especially those airports with smaller degrees and in remote regions. Though the disconnections of such regions would not severely reduce the overall network efficiency nor the robustness, it would significantly damage the local and regional livelihood not only in the air transportation industry-related fields but also in local manufacturing sectors that depend on air transportation. In the meantime, it is also crucial for the policymakers and investors to take, scrutinize, and reevaluate the role of the airports that have higher centrality values. Additionally, we find that the network is robust against the edge removal strategies, and the closure of the airports with high centrality values, such as Memphis, Louisville, Chicago, and Anchorage, would incur severe damage to the network structure integrity and performance. Third, as seen in this study, both the efficiency and robustness of the largest community structures have increased, and thus in the future adjustment of network structure, decision-makers should direct more attention to the development of regional hubs to foster network integrity.

\section{Data Availability}

The data in this research can be obtained at https://www.bts. gov/browse-statistical-products-and-data, the Bureau of Transportation Statistics, and to implement the analysis in 
this study, all the tools employed in this study, including QGIS, python 3.8.8, and Gephi, are all freely available online.

\section{Conflicts of Interest}

The authors declare that they have no conflicts of interest.

\section{Acknowledgments}

This study was funded by the National Natural Science Foundation of China (NSFC) no. 41871162.

\section{References}

[1] IATA, “IATA annual review 2020," 2020, https://www.iata. org/contentassets/c81222d96c9a4e0bb4ff6ced0126f0bb/iataannual-review-2020.pdf.

[2] S. Boccaletti, V. Latora, Y. Moreno, M. Chavez, and D. Hwang, "Complex networks: structure and dynamics," Physics Reports, vol. 424, no. 4-5, pp. 175-308, 2006.

[3] A. Bombelli, B. F. Santos, and L. Tavasszy, "Analysis of the air cargo transport network using a complex network theory perspective," Transportation Research Part E: Logistics and Transportation Review, vol. 138, Article ID 101959, 2020.

[4] O. Lordan, J. M. Sallan, and P. Simo, "Study of the topology and robustness of airline route networks from the complex network approach: a survey and research agenda," Journal of Transport Geography, vol. 37, pp. 112-120, 2014.

[5] X. Zeng, X. Tang, and K. Jiang, "Empirical study of Chinese airline network structure based on complex network theory," Journal of Transportation Systems Engineering and Information Technology, vol. 11, no. 6, pp. 175-181, 2011.

[6] J. D. Kasarda and J. D. Green, "Air cargo as an economic development engine: a note on opportunities and constraints," Journal of Air Transport Management, vol. 11, no. 6, pp. 459-462, 2005.

[7] R. Klophaus, R. Conrady, and F. Fichert, "Low cost carriers going hybrid: evidence from Europe," Journal of Air Transport Management, vol. 23, pp. 54-58, 2012.

[8] M. Mercan and E. Karakaya, "Energy consumption, economic growth and carbon emission: dynamic panel cointegration analysis for selected OECD countries," Procedia Economics and Finance, vol. 23, pp. 587-592, 2015.

[9] H. Küçükönal and G. Sedefoğlu, "The causality analysis of air transport and socio-economics factors: the case of OECD countries," Transportation Research Procedia, vol. 28, pp. 16-26, 2017.

[10] L. Budd and S. Ison, "The role of dedicated freighter aircraft in the provision of global airfreight services," Journal of Air Transport Management, vol. 61, pp. 34-40, 2017.

[11] L. E. C. da Rocha, "Structural evolution of the Brazilian airport network," Journal of Statistical Mechanics: Theory and Experiment, vol. 2009, no. 4, Article ID P04020, 2009.

[12] W.-B. Du, X.-L. Zhou, O. Lordan, Z. Wang, C. Zhao, and Y.-B. Zhu, "Analysis of the Chinese airline network as multilayer networks," Transportation Research Part E: Logistics and Transportation Review, vol. 89, pp. 108-116, 2016.

[13] R. Guimerà and L. A. N. Amaral, "Modeling the world-wide airport network," The European Physical Journal B (EPJ B)Condensed Matter, vol. 38, no. 2, pp. 381-385, 2004.

[14] R. Guimera, S. Mossa, A. Turtschi, and L. A. N. Amaral, "The worldwide air transportation network: anomalous centrality, community structure, and cities' global roles," Proceedings of the National Academy of Sciences, vol. 102, no. 22, pp. 7794-7799, 2005.

[15] C. Hong, J. Zhang, X.-B. Cao, and W.-B. Du, "Structural properties of the Chinese air transportation multilayer network," Chaos, Solitons \& Fractals, vol. 86, pp. 28-34, 2016.

[16] T. Jia, K. Qin, and J. Shan, "An exploratory analysis on the evolution of the US airport network," Physica A: Statistical Mechanics and its Applications, vol. 413, pp. 266-279, 2014.

[17] A. Reynolds-Feighan, "Characterisation of airline networks: a North American and European comparison," Journal of Air Transport Management, vol. 16, no. 3, pp. 109-120, 2010.

[18] L. Sanders, "Models in spatial analysis," in Models in Spatial AnalysisESRI, 2010.

[19] M. Sapre and N. Parekh, "Analysis of centrality measures of airport network of India," Computer Science, vol. 6744, 2011.

[20] A. Reynolds-Feighan, "Traffic distribution in low-cost and full-service carrier networks in the US air transportation market," Journal of Air Transport Management, vol. 7, no. 5, pp. 265-275, 2001.

[21] A. Zhang, Y. Wan, and H. Yang, "Impacts of high-speed rail on airlines, airports and regional economies: a survey of recent research," Transport Policy, vol. 81, pp. A1-A19, 2019.

[22] A. Barrat, M. Barthélemy, R. Pastor-Satorras, and A. Vespignani, "The architecture of complex weighted networks," Proceedings of the National Academy of Sciences, vol. 101, no. 11, pp. 3747-3752, 2004.

[23] V. Colizza, A. Barrat, M. Barthélemy, and A. Vespignani, "The role of the airline transportation network in the prediction and predictability of global epidemics," Proceedings of the National Academy of Sciences, vol. 103, no. 7, pp. 2015-2020, 2006.

[24] C. Zhao, C. Xiu, and G. Yu, "FedEx and UPS network structure and accessibility analysis based on complex network theory," Complexity, vol. 2021, Article ID 6682670, 15 pages, 2021.

[25] L. Demetrius and T. Manke, "Robustness and network evolution-an entropic principle," Physica A: Statistical Mechanics and its Applications, vol. 346, no. 3-4, pp. 682-696, 2005.

[26] N. Ahmad and S. Derrible, "An information theory based robustness analysis of energy mix in US States," Energy Policy, vol. 120, pp. 167-174, 2018.

[27] M. Amini and M. Almassalkhi, "Trading off robustness and performance in receding horizon control with uncertain energy resources," in Proceedings of the 2018 Power Systems Computation Conference (PSCC), Dublin, Ireland, June 2018.

[28] R. Burian, M. Gontijo, and H. Alvarez, "Robustness and reliability in smart grid solutions," in Proceedings of the 2019 IEEE 7th International Conference On Smart Energy Grid Engineering (SEGE), Ontario, Canada, August 2019.

[29] E. Borgomeo, M. Mortazavi-Naeini, J. W. Hall, and B. P. Guillod, "Risk, robustness and water resources planning under uncertainty," Earth's Future, vol. 6, no. 3, pp. 468-487, 2018.

[30] D. Jung, D. Kang, J. H. Kim, and K. Lansey, "Robustnessbased design of water distribution systems," Journal of Water Resources Planning and Management, vol. 140, no. 11, Article ID 04014033, 2014.

[31] G. Pronk, S. Stofberg, T. Van Dooren et al., "Increasing water system robustness in The Netherlands: potential of crosssectoral water reuse," Water Resources Management, vol. 15, 2021.

[32] E. Rozos, C. Makropoulos, and D. Butler, "Design robustness of local water-recycling schemes," Journal of Water Resources Planning and Management, vol. 136, no. 5, pp. 531-538, 2010. 
[33] J. P. Cárdenas, M. L. Mouronte, L. G. Moyano, M. L. Vargas, and R. M. Benito, "On the robustness of Spanish telecommunication networks," Physica A: Statistical Mechanics and its Applications, vol. 389, no. 19, pp. 4209-4216, 2010.

[34] S. Eldh and D. Sundmark, "Robustness testing of mobile telecommunication systems: a case study on industrial practice and challenges," in Proceedings of the 2012 IEEE Fifth International Conference on Software Testing, Verification and Validation, pp. 895-900, Montreal, Canada, April 2012.

[35] Y. Fan, F. Zhang, S. Jiang et al., "Dynamic robustness analysis for subway network with spatiotemporal characteristic of passenger flow," IEEE Access, vol. 8, pp. 45544-45555, 2020.

[36] E. Frutos Bernal and A. Martín del Rey, "Study of the structural and robustness characteristics of madrid metro network," Sustainability, vol. 11, no. 12, Article ID 3486, 2019.

[37] Y. Yang, Y. Liu, M. Zhou, F. Li, and C. Sun, "Robustness assessment of urban rail transit based on complex network theory: a case study of the Beijing Subway," Safety Science, vol. 79, pp. 149-162, 2015.

[38] O. Cats and E. Jenelius, "Planning for the unexpected: the value of reserve capacity for public transport network robustness," Transportation Research Part A: Policy and Practice, vol. 81, pp. 47-61, 2015.

[39] R. A. El-Rashidy and S. M. Grant-Muller, "An assessment method for highway network vulnerability," Journal of Transport Geography, vol. 34, pp. 34-43, 2014.

[40] J. Monteil, R. Billot, J. Sau, F. Armetta, S. Hassas, and N.-E. E. Faouzi, "Cooperative highway traffic," Transportation Research Record: Journal of the Transportation Research Board, vol. 2391, no. 1, pp. 1-10, 2013.

[41] N. Zhang and A. Alipour, "Multi-scale robustness model for highway networks under flood events," Transportation Research Part D: Transport and Environment, vol. 83, Article ID 102281, 2020.

[42] P. Angeloudis, K. Bichou, and M. G. Bell, "Security and reliability of the liner container-shipping network: analysis of robustness using a complex network framework," in Risk Management in Port Operations, Logistics and Supply Chain Securitypp. 129-140, Informa Law from Routledge, Oxfordshire, UK, 2013.

[43] P. Peng, S. Cheng, J. Chen et al., "A fine-grained perspective on the robustness of global cargo ship transportation networks," Journal of Geographical Sciences, vol. 28, no. 7, pp. 881-889, 2018.

[44] N. Wang, N. Wu, L. L. Dong, H. K. Yan, and D. Wu, "A study of the temporal robustness of the growing global containershipping network," Scientific Reports, vol. 6, no. 1, pp. 34217-10, 2016.

[45] L. Dall'Asta, A. Barrat, M. Barthélemy, and A. Vespignani, "Vulnerability of weighted networks," Journal of Statistical Mechanics: Theory and Experiment, vol. 2006, no. 4, Article ID P04006, 2006.

[46] O. Lordan, J. M. Sallan, N. Escorihuela, and D. GonzalezPrieto, "Robustness of airline route networks," Physica A: Statistical Mechanics and its Applications, vol. 445, pp. 18-26, 2016.

[47] O. Lordan, J. M. Sallan, P. Simo, and D. Gonzalez-Prieto, "Robustness of the air transport network," Transportation Research Part E-Logistics And Transportation Review, vol. 68, 2014.

[48] K.-C. Pien, K. Han, W. Shang, A. Majumdar, and W. Ochieng, "Robustness analysis of the European air traffic network," Transportmetrica: Transportation Science, vol. 11, no. 9, pp. 772-792, 2015.
[49] K. L. Clark, U. Bhatia, E. A. Kodra, and A. R. Ganguly, "Resilience of the U.S. National airspace system Airport network," IEEE Transactions on Intelligent Transportation Systems, vol. 19, no. 12, pp. 3785-3794, 2018.

[50] Y. Chen, J. Wang, and F. Jin, "Robustness of China's air transport network from 1975 to 2017," Physica A: Statistical Mechanics and its Applications, vol. 539, Article ID 122876, 2020.

[51] G. Ren, "Robustness analysis of air route network based on topology potential and relative entropy methods," Journal of Advanced Transportation, vol. 2021, Article ID 5527423, 11 pages, 2021.

[52] S. Dunn and S. M. Wilkinson, "Increasing the resilience of air traffic networks using a network graph theory approach," Transportation Research Part E: Logistics and Transportation Review, vol. 90, pp. 39-50, 2016.

[53] M. Janić, "Modeling the resilience of an airline cargo transport network affected by a large scale disruptive event," Transportation Research Part D: Transport and Environment, vol. 77, pp. 425-448, 2019.

[54] D. Warnock-Smith and P. Morrell, "Air transport liberalisation and traffic growth in tourism-dependent economies: a case-history of some US-Caribbean markets," Journal of Air Transport Management, vol. 14, no. 2, pp. 82-91, 2008.

[55] G. Burghouwt and J. G. de Wit, "In the wake of liberalisation: long-term developments in the EU air transport market," Transport Policy, vol. 43, pp. 104-113, 2015.

[56] M. Abate, "Economic effects of air transport market liberalization in Africa," Transportation Research Part A: Policy and Practice, vol. 92, pp. 326-337, 2016.

[57] P. Malighetti, G. Martini, R. Redondi, and D. Scotti, "Air transport networks of global integrators in the more liberalized Asian air cargo industry," Transport Policy, vol. 80, pp. 12-23, 2019.

[58] A. D. Cristea, R. Hillberry, and A. Mattoo, "Open skies over the Middle East," The World Economy, vol. 38, no. 11, pp. 1650-1681, 2015.

[59] R. Piermartini and L. Rousová, "The sky is not flat: how discriminatory is the access to international air services?" American Economic Journal: Economic Policy, vol. 5, no. 3, pp. 287-319, 2013.

[60] V. Benezech, L. Martinez, and J. Kauppila, "Assessing the long-term impact of air liberalization on international air passenger demand," 2016.

[61] N. Avogadro, P. Malighetti, R. Redondi, and A. Salanti, "A tale of airline competition: when full-service carriers undercut low-cost carriers fares," Journal of Air Transport Management, vol. 92, 2021.

[62] M. Ben Abda, P. P. Belobaba, and W. S. Swelbar, "Impacts of LCC growth on domestic traffic and fares at largest US airports," Journal of Air Transport Management, vol. 18, no. 1, pp. 21-25, 2012.

[63] F. Dobruszkes and J. Wang, "Developing a low-cost airline in a semi-protected regime: comparing China to Europe and the US," Journal of Transport Geography, vol. 77, pp. 48-58, 2019.

[64] Y. Jiang, B. Yao, L. Wang, T. Feng, and L. Kong, "Evolution trends of the network structure of Spring Airlines in China: a temporal and spatial analysis," Journal of Air Transport Management, vol. 60, pp. 18-30, 2017.

[65] W. Ma, A. Zhang, Y. Zhang, and S. Xu, "The growing influence of low-cost carriers in Northeast Asia and its implications for a regional single aviation market," Journal of Air Transport Management, vol. 91, Article ID 101994, 2021. 
[66] Z. Xu and R. Harriss, "Exploring the structure of the U.S. intercity passenger air transportation network: a weighted complex network approach," Geojournal, vol. 73, no. 2, pp. 87-102, 2008.

[67] Y. Zhou, J. Wang, and G. Q. Huang, "Efficiency and robustness of weighted air transport networks," Transportation Research Part E: Logistics and Transportation Review, vol. 122, pp. 14-26, 2019.

[68] U. Brandes, "A faster algorithm for betweenness centrality," Journal of Mathematical Sociology, vol. 25, no. 2, pp. 163-177, 2001.

[69] J. Kauffman, A. Kittas, L. Bennett, and S. Tsoka, "DyCoNet: a Gephi plugin for community detection in dynamic complex networks," PLoS One, vol. 9, no. 7, Article ID e101357, 2014.

[70] M. E. J. Newman, "The structure and function of complex networks," SIAM Review, vol. 45, no. 2, pp. 167-256, 2003.

[71] V. D. Blondel, J.-L. Guillaume, R. Lambiotte, and E. Lefebvre, "Fast unfolding of communities in large networks," Journal of Statistical Mechanics: Theory and Experiment, vol. 2008, no. 10, Article ID P10008, 2008.

[72] S. L. Cutter, J. A. Ahearn, B. Amadei et al., "Disaster resilience: a national imperative," Environment: Science and Policy for Sustainable Development, vol. 55, no. 2, pp. 25-29, 2013.

[73] O. Lordan and M. Albareda-Sambola, "Exact calculation of network robustness," Reliability Engineering \& System Safety, vol. 183, pp. 276-280, 2019.

[74] C. M. Schneider, A. A. Moreira, J. S. Andrade, S. Havlin, and H. J. Herrmann, "Mitigation of malicious attacks on networks," Proceedings of the National Academy of Sciences, vol. 108, no. 10, pp. 3838-3841, 2011.

[75] V. Latora and M. Marchiori, "Efficient behavior of smallworld networks," Physical Review Letters, vol. 87, no. 19, Article ID 198701, 2001.

[76] A.-L. Barabási and E. Bonabeau, "Scale-free networks," Scientific American, vol. 288, no. 5, pp. 60-69, 2003.

[77] D. J. Watts and S. H. Strogatz, "Collective dynamics of 'smallworld' networks," Nature, vol. 393, pp. 440-2, 1998.

[78] R. K. Merton, "The Matthew effect in science," Science, vol. 159, no. 3810, pp. 56-63, 1968. 